\title{
Modified candy-plug device for aneurysmal false lumen occlusion in chronic type B aortic dissection
}

\author{
I-Hui Wu, MD, PhD, ${ }^{\mathrm{a}}$ Chih-Yang Chan, $\mathrm{MD}, \mathrm{PhD},{ }^{\mathrm{a}}$ Chien-Ming Luo, MD, ${ }^{\mathrm{b}}$ and \\ Shoei-Shen Wang, MD, PhD, ${ }^{a, c}$ Taipei, Hsin-Chu, and New Taipei City, Taiwan
}

\footnotetext{
From the a Division of Cardiovascular Surgery, Department of Surgery, National Taiwan University Hospital, Taipei, Taiwan; ${ }^{b}$ Department of Surgery, National Taiwan University Hospital Hsin-Chu Branch, Hsin-Chu, Taiwan; and ${ }^{\mathrm{c}}$ Department of Surgery, Fu Jen Catholic University Hospital, and Fu Jen Catholic University College of Medicine, New Taipei City, Taiwan.

Disclosures: Authors have nothing to disclose with regard to commercial support.

Received for publication Oct 11, 2017; revisions received Dec 6, 2017; accepted for publication Dec 20, 2017; available ahead of print Feb 16, 2018.

Address for reprints: I-Hui Wu, MD, PhD, Cardiovascular Section, Department of Surgery, National Taiwan University Hospital, No. 7, Chung-Shan S. Rd, Taipei, Taiwan (E-mail: aaronihuiwu@ gmail.com).

J Thorac Cardiovasc Surg 2018;155:1970-2

$0022-5223 / \$ 36.00$

Copyright (c) 2018 by The American Association for Thoracic Surgery

https://doi.org/10.1016/j.jtcvs.2017.12.122
}

Uf Supplemental material is available online.

$\square$ Video clip is available online.

In patients with chronic type B aortic dissection aneurysm (CTBAD) with false lumen (FL) aneurysm, thoracic endovascular aneurysm repair (TEVAR) has been increasingly performed by covering the proximal entry alone. However, one of the most common delayed complications is continued FL perfusion with aneurysmal

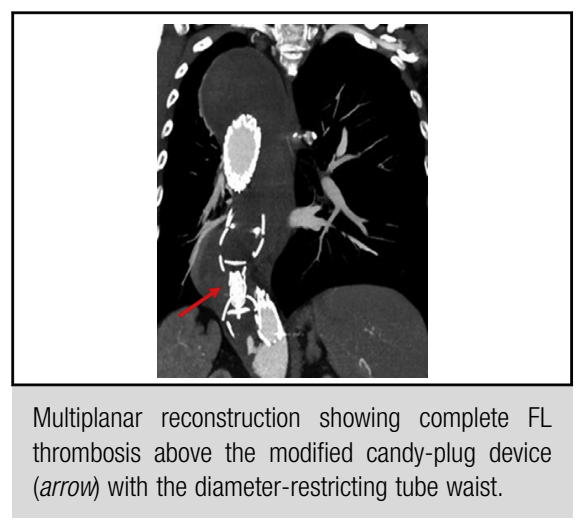

Central Message

The candy-plug technique facilitates complete false occlusion by prohibiting distal FL backflow. The current modification of a candyplug waist makes this technique more precise and durable.

See Editorial Commentary page 1973.

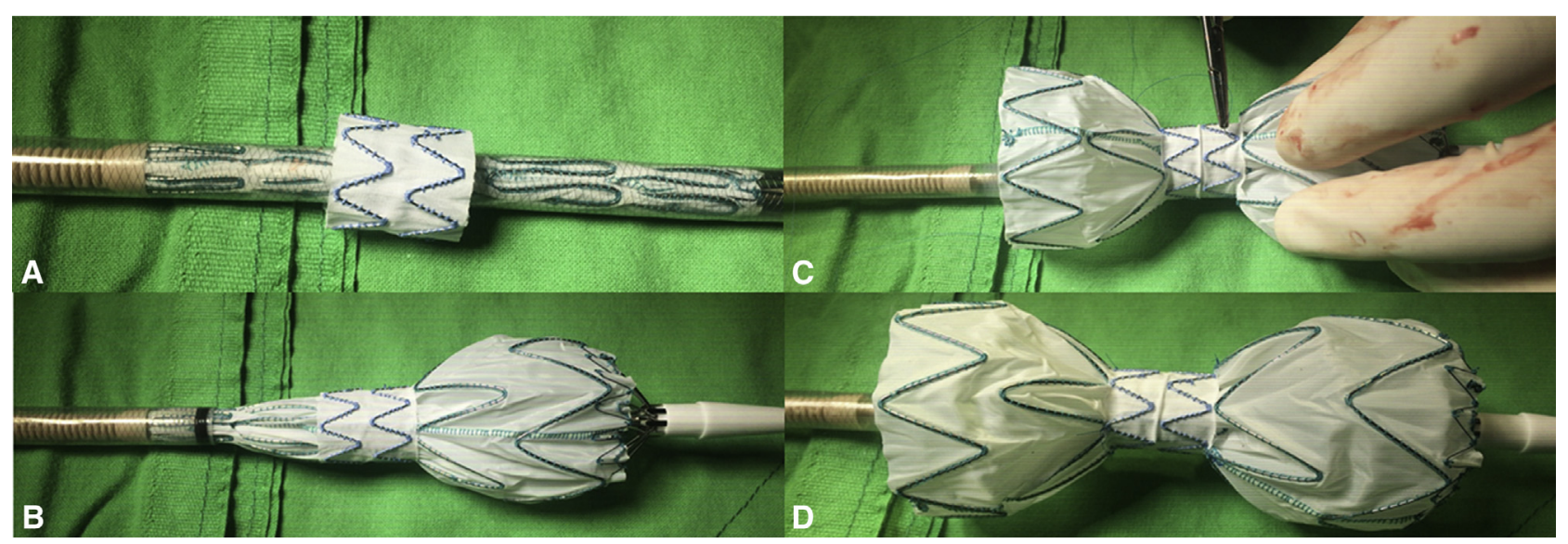

FIGURE 1. A, Trimmed iliac limb $(2 \mathrm{~cm})$ was positioned in the middle of the thoracic SG and (B) telescoping the thoracic SG. C, Stay sutures were placed at both sides of the iliac limb. D, The diameter-restricting tube waist in the middle of the SG was wide enough to retrieve the dilator tip of the delivery system. 


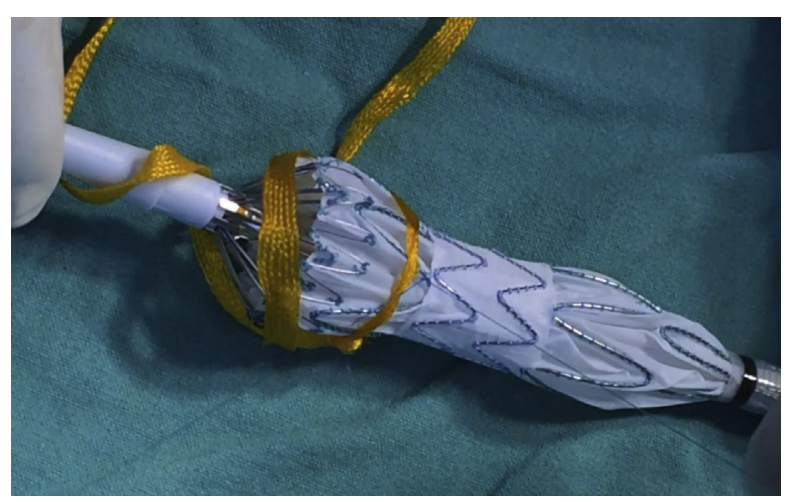

VIDEO 1. Side-table preparation of physician-modified candy-plug device. Video available at: http://www.jtcvsonline.org/article/S00225223(18)30056-4/fulltext.

Techniques involving a homemade candy-plug device with a diameter-reducing suture to prevent persistent flow from distal fenestrations have been reported. ${ }^{3,4}$ In the present report, we describe a novel technique that involves telescoping a short segment of the iliac limb as a substitution for the diameter-reducing suture outside the thoracic stent-graft (SG) used as a customized candy plug for FL occlusion.

\section{TECHNIQUE}

The modified technique was demonstrated in a 64-year-old patient with CTBAD undergoing TEVAR with persistent perfusion and enlargement of FL at 6-month follow-up (Figure E1). The patient was subsequently scheduled to undergo the exclusion of the distal fenestration flow to the proximal FL by using the physician-modified candy-plug device. A $44 \times 100-\mathrm{mm}$ Medtronic Valiant Captivia FreeFlo straight thoracic device (Medtronic Vascular, Santa Rosa, Calif) and a $16 \times 82-\mathrm{mm}$ Endurant iliac limb (Medtronic, Minneapolis, Minn) were placed on the side table. This iliac limb was shortened to $20 \mathrm{~mm}$ and positioned outside the middle of the thoracic endoprosthesis (Figure 1, A). This shortened iliac limb was telescoped outside the partially unloaded thoracic SG (Figure 1, B). The iliac limb was subsequently sutured to the SG at each quarter clockwise position with 7-0 Prolene for fixation (Figure 1,C). This procedure gives the $\mathrm{SG}$ a wrapped candy shape, with a waist sufficiently large to retrieve the tapering tip (Figure 1,D). The customized SG was reloaded and prepared in a standard manner (Video 1). Both the true lumen (TL) and FL were catheterized after the cutdown of femoral access on both sides. An extra-stiff Lunderquist wire was positioned first in the ascending aorta through the TL via the left femoral access, and another pigtail catheter was positioned in the FL through the fenestration below the renal a- rtery via the right femoral access. We first relined the TL with a $34 \times 150-\mathrm{mm}$ Medtronic Valiant Captivia ClosedWeb straight SG positioned in the TL, and the candy-plug device was positioned parallel to the TL-extension SG in the FL (Figure 2, A). A 22-mm Amplatzer vascular plug II (St Jude Medical, St Paul, Minn) was then placed in the waist of the device to complete the occlusion (Figure 2, B). Follow-up computed tomography angiography at 6 months revealed no endoleaks and showed complete FL thrombosis above the candy-plug device (Figure 2, $C$ and $D$ ).

\section{COMMENT}

In patients with CTBAD with persistent FL perfusion from distal fenestrations after TEVAR, complete FL exclusion has been advocated by paving the fenestrated or branched SG down to the iliac arteries to occlude all the communications between the TL and the FL. However, the intricacy of this advanced intervention and risk of spinal cord ischemia associated with covering all the segmental arteries during the treatment prevent its application in clinical practice. ${ }^{5}$

Several modifications of the candy-plug technique have been published. However, most of these techniques require placing a vascular plug in the waist of the candy-plug SG with only a diameter-restricting suture to constrain the waist. The long-term durability of the suture in sustaining the waist of a self-expandable SG is questionable, and the landing zone of the vascular plug with the SG relies on the diameter of only 1 suture. In this technique, we replace the constraining suture with an iliac limb extender. This extender facilitates more precise measurement of the waist diameter and ensures a more lengthy zone and more durable support than does a constraining suture for the vascular plug to seal the restricted opening of the SG. The candyplug size was $10 \%$ to $30 \%$ larger than the averaged diameter of FL. It was deployed parallel to the SG in the TL at $1 \mathrm{~cm}$ above the celiac axis. ${ }^{5}$ It could avoid narrowing the TL and protect the intimal flap injury from the candy-plug device. The gradual expansion of both TL and FL SGs might further seal retrograde endoleaks if any exist. Small limb diameter might prevent the nosecone retraction through the waist. In our study, we suggested a 16-mm iliac limb to accommodate the commercially available largest vascular plug (Figures $\mathrm{E} 2$ and E3).

\section{CONCLUSIONS}

The candy-plug technique facilitates complete FL occlusion by prohibiting distal FL backflow. The current modification for developing a candy-plug waist makes 


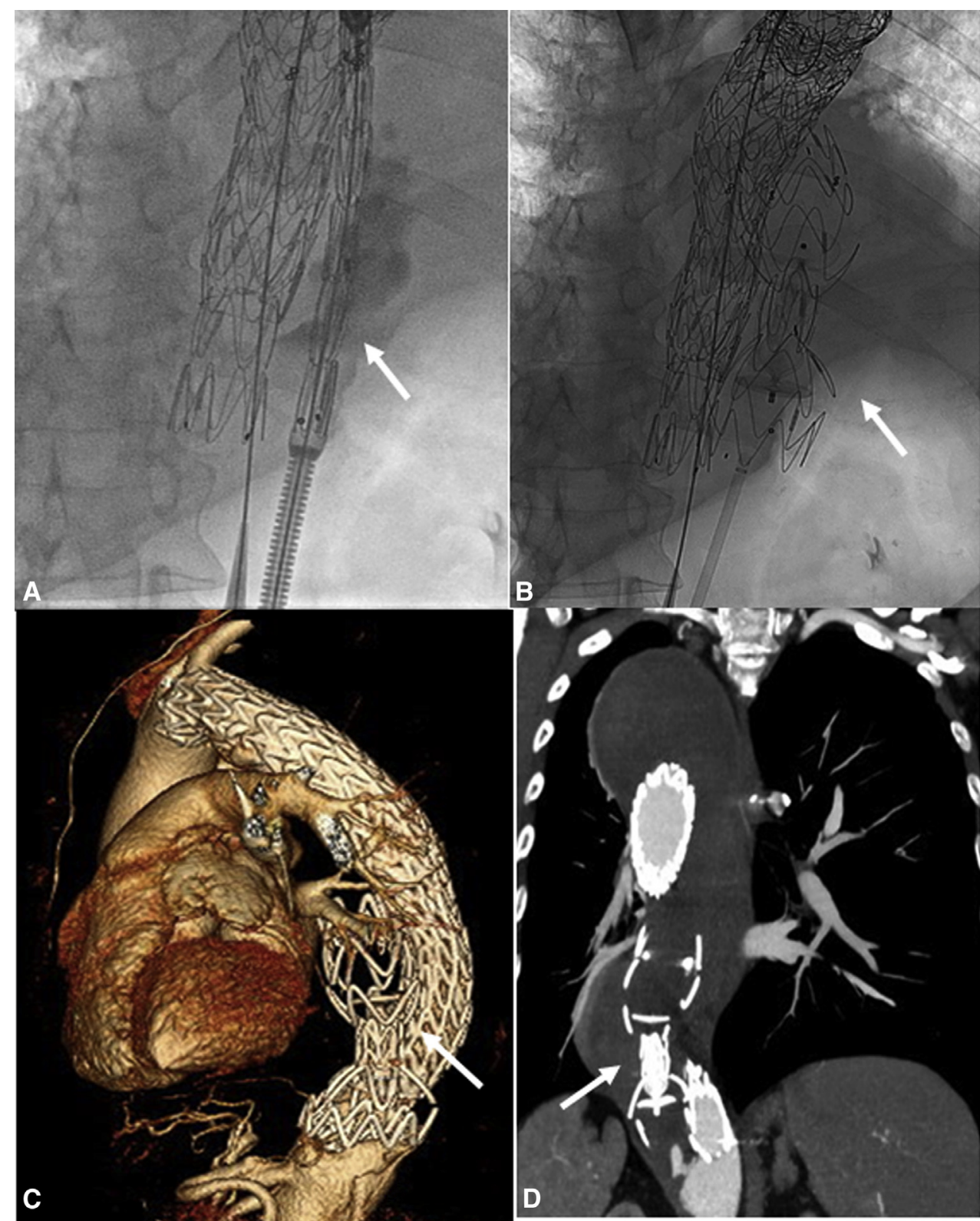

FIGURE 2. A, Modified candy-plug device (arrow) was positioned parallel to the thoracic SG in the TL. B, A 22-mm Amplatzer (St Jude Medical, St Paul, Minn) vascular plug II (arrow) was placed in the tube waist of the modified device. C, Volume rendering revealing the position of the large-diameter vascular plug in the FL (arrow). D, Multiplanar reconstruction showing complete FL thrombosis above the modified candy-plug device (arrow) without retrograde endoleaks.

this technique more precise and durable. Further follow-up studies are necessary.

\section{References}

1. Thrumurthy SG, Karthikesalingam A, Patterson BO, Holt PJE, Hinchliffe RJ, Loftus IM, et al. A systematic review of mid-term outcomes of thoracic endovascular repair (TEVAR) of chronic type B aortic dissection. Eur J Vasc Endovasc Surg. 2011;42:632-47.

2. Chemelli-Steingruber I, Chemelli A, Strasak A, Hugl B, Hiemetzberger R, Jaschke W, et al. Endovascular repair or medical treatment of acute type B aortic dissection? A comparison. Eur J Radiol. 2010;73:175-80.
3. Kolbel T, Lohrenz C, Kieback A, Diener H, Debus ES, LarenaAvellaneda A. Distal false lumen occlusion in aortic dissection with a homemade extra-large vascular plug: the candy-plug technique. J Endovasc Ther. 2013;20:484-9.

4. Ogawa Y, Nishimaki H, Chiba K, Murakami K, Sakurai Y, Fujiwara K, et al. Candy-plug technique using an excluder aortic extender for distal occlusion of a large false lumen aneurysm in chronic aortic dissection. J Endovasc Ther. 2016; 23:483-6.

5. Kolbel T, Rohlffs F, Debus ES, Tsilimparis N. Polytetrafluoroethylene excludes the false lumen: expanding material options for the candy-plug technique. J Endovasc Ther. 2016;23:487-8. 


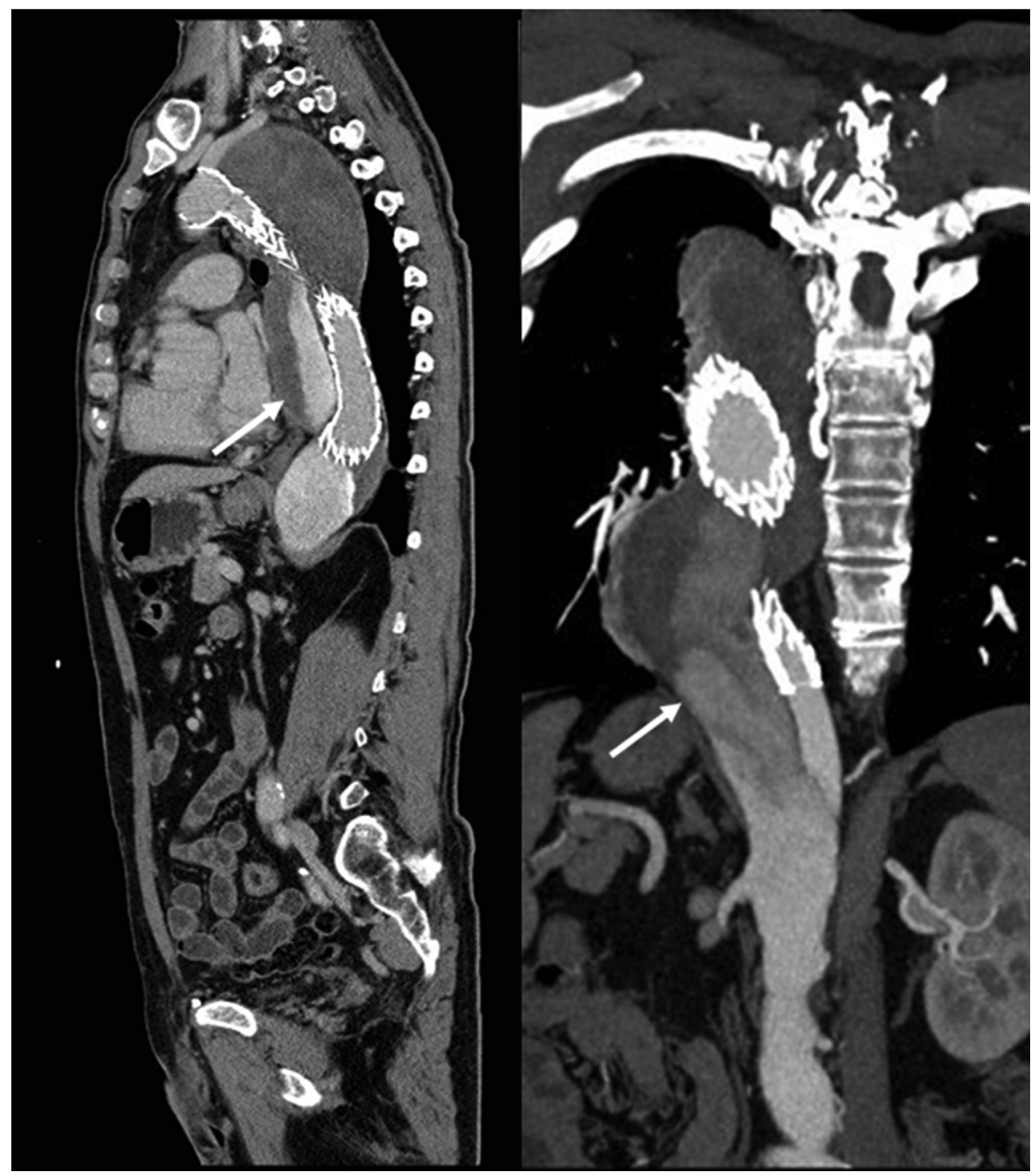

FIGURE E1. Multiplanar reconstruction showing persistent retrograde perfusion of the proximal FL (arrow) from the distal fenestration. 


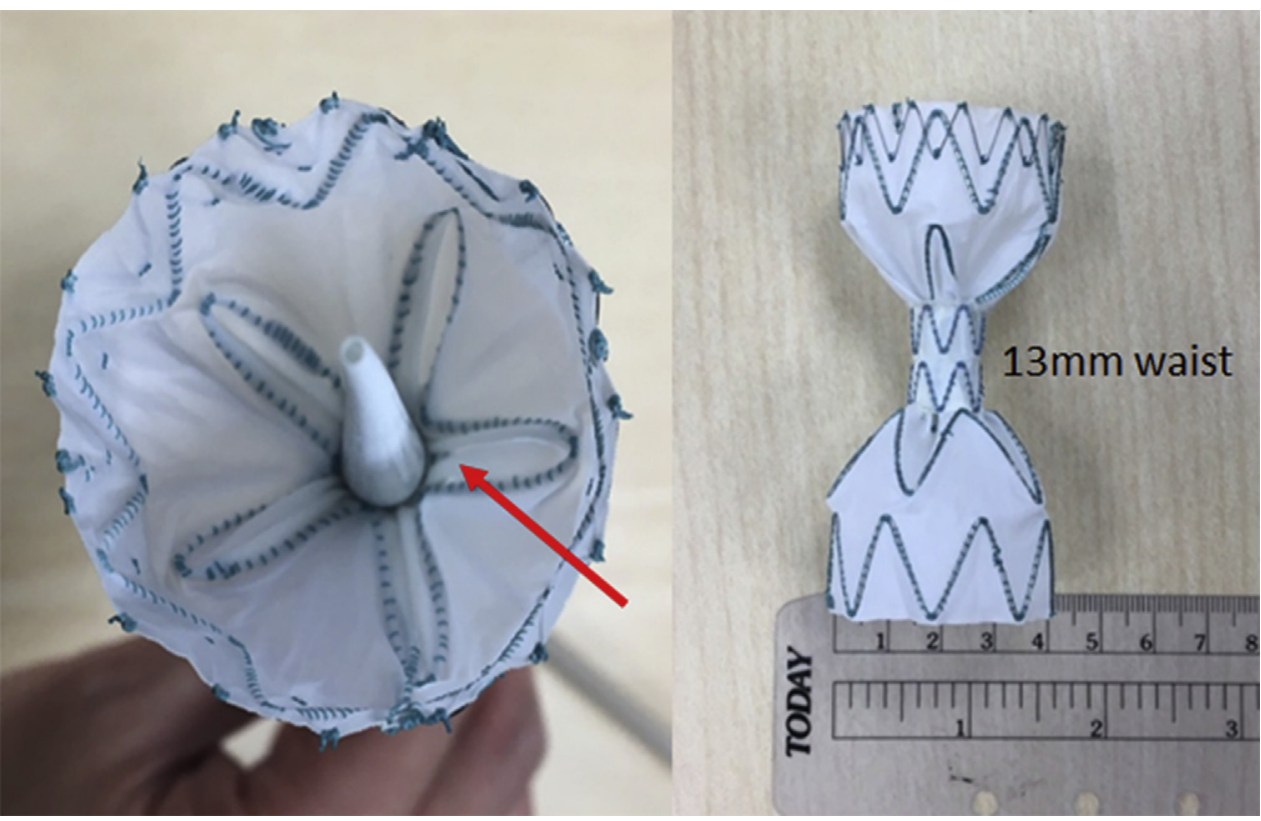

FIGURE E2. The 13-mm diameter-restricting tube waist in the middle of the SG was not wide enough (arrow) to retrieve the dilator tip of the delivery system.

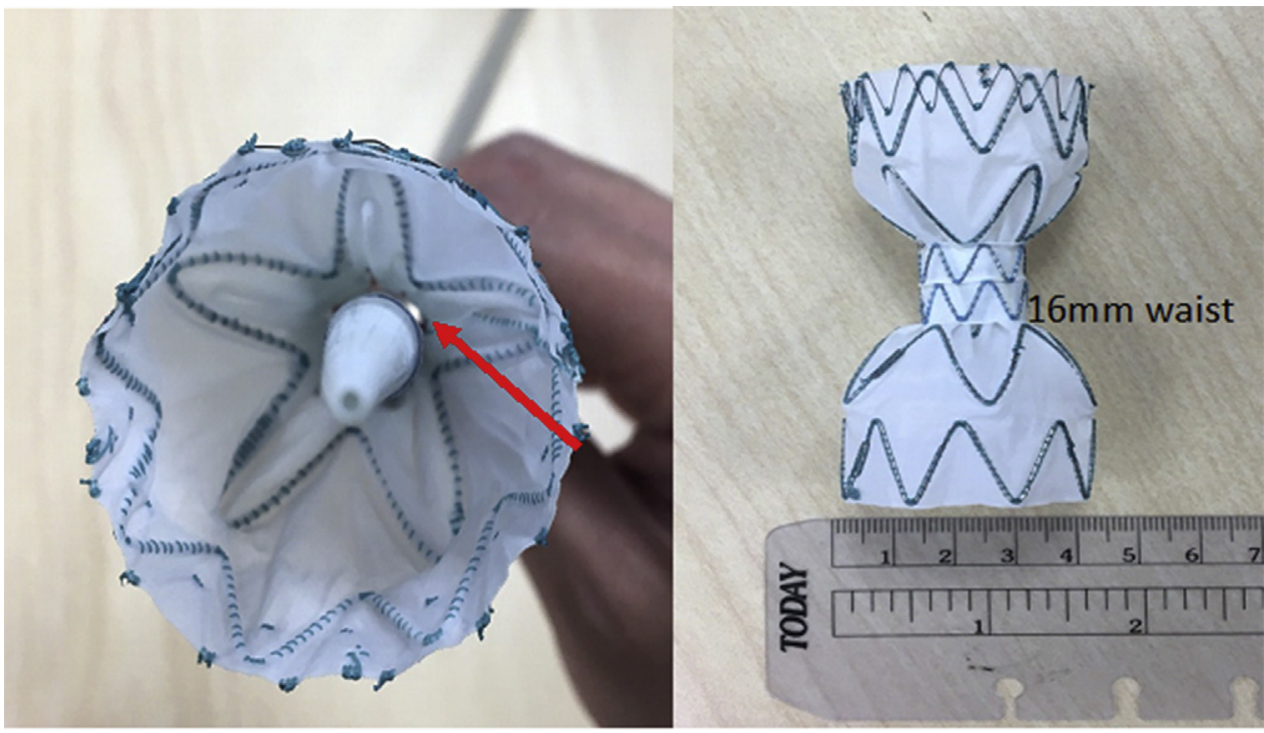

FIGURE E3. The 16-mm diameter-restricting tube waist in the middle of the SG was wide enough (arrow) to retrieve the dilator tip of the delivery system. 